solution such that $\varphi$ has a logarithmic singularity at the point $x_{0}, y_{0} ; \psi$ (of course) has an "arc tan behavior" at this point. It is immediately evident that we are looking for the Green's function of Eq. (5), say for the region $y>0$. To find this, we merely put a spurious. non-homogeneous term $N(x, s)$ on the right side of (5), define $\bar{N}$ as we did $\bar{\chi}$, and operate as before.

This time we find

$$
\left(\xi^{2}+\alpha^{2}\right) \bar{\chi}=\bar{N}
$$

if we allow the boundary contributions to vanish. The inversion of Eq. (17) leads to

$$
\chi=\int_{-\infty}^{\infty} \int_{0}^{\infty} s_{0} N\left(x_{0}, s_{0}\right) \int_{-\infty}^{\infty} \int_{0}^{\infty} \frac{\alpha e^{i \xi\left(x-x_{0}\right)} J_{2 / 3}(\alpha s)}{\xi^{2}+\alpha^{2}} J_{2 / 3}\left(\alpha s_{0}\right) d \xi d \alpha d x_{0} d s_{0}
$$

where the double integral over $\xi$ and $\alpha$ must define the Green's function. Thus, we write

$$
\begin{aligned}
G\left(x, x_{0} ; s, s_{0}\right) & =\int_{0}^{\infty} e^{-\alpha\left|x-x_{0}\right|} J_{2 / 3}(\alpha s) J_{2 / 3}\left(\alpha s_{0}\right) d \alpha \\
& =A s^{-1 / 2} Q_{1 / 6}\left(\frac{\left(x-x_{0}\right)^{2}+s^{2}+s_{0}^{2}}{2 s s_{0}}\right)
\end{aligned}
$$

where $A$ is some constant.

The corresponding solutions $\varphi$ and $\psi$ are

$$
\begin{aligned}
& \varphi=y^{1 / 4} Q_{1 / 6}\left(\frac{\left(x-x_{0}\right)^{2}+y^{3}+y_{0}^{3}}{2\left(y y_{0}\right)^{3 / 2}}\right) \\
& \psi=\int^{x}\left(\varphi_{\nu} / y\right) d x
\end{aligned}
$$

It is interesting to note that the stream function $\psi$ is closely related to one found by Weinstein. ${ }^{4}$

If we had formally used $J_{-2 / 3}$ again, the index $1 / 6$ appearing in the Legendre function would be replaced by $-7 / 6$. Either solution may readily be continued into the hyperbolic domain.

\title{
THE LOST SOLUTIONS IN AXIALLY SYMMETRIC IRROTATIONAL COMPRESSIBLE FLUID FLOW*
}

\section{By H. J. STEWART (California Institute of Technology)}

1. Since the two dimensional potential equation for the irrotational flow of a compressible fluid may be linearized by a Legendre transformation from the physical plane to the velocity (hodograph) plane, this linearizing transformation is usually one of the first steps taken in the study of two dimensional compressible fluid flows. This trans-

${ }^{4}$ Weinstein, A., On axially symmetric flows, Q. Appl. Math. 5, 429 (1948).

*Received Jan. 12, 1948. 
formation is degenerate for any flow for which one velocity component is a function of the other velocity component. In this case the entire physical plane maps into a line in the hodograph plane. Such a solution to the potential flow equation is called a "lost" solution because the Legendre transformation to the hodograph plane is not valid in these circumstances and cannot be used to investigate such a flow. In the two dimensional supersonic theory this type of flow, generally called Prandtl-Meyer flow, ${ }^{1}$ is of great importance since it includes the expanding flow around a corner or over any convexly curved surface. It is also important in the design of supersonic wind tunnel nozzles since the matching flow between a two dimensional source flow (representing the initial expansion near the nozzle throat) and the rectilinear flow in the working section is of the Prandtl-Meyer type.

Because of the importance of the lost solution in the two dimensional case, the question of the existence and nature of similarly degenerate flows in three dimensional flow problems is of considerable interest. The complete solution to this question for one class of three dimensional flows, axially symmetric flows, can be obtained by a very simple extension of a method used by Bateman ${ }^{2}$ in treating the two dimensional problem.

2. Consider an axially symmetric irrotational compressible fluid flow with $x$ being the distance along the axis of symmetry and $y$ being the radial distance from the axis. Let $u$ and $v$ be the velocity components in the $x$ and $y$ directions respectively, and let $\rho$ be the density of the fluid. The functional relation between the velocity components may be written in a parametric form as

$$
u=A(s), \quad v=B(s), \quad \rho=F(s),
$$

where $F(s)$ may be expressed in terms of $A(s)$ and $B(s)$ by the momentum integral and the parameter $s=s(x, y)$ represents the lines of constant velocity in the physical plane. Since the flow is irrotational,

$$
\frac{\partial v}{\partial x}=\frac{\partial u}{\partial y}
$$

or

$$
B^{\prime}(s) \frac{\partial s}{\partial x}=A^{\prime}(s) \frac{\partial s}{\partial y} .
$$

This shows that the lines of constant $s$ must be straight lines of the type

$$
x A^{\prime}(s)+y B^{\prime}(s)=C^{\prime}(s),
$$

where $C^{\prime}(s)$ is an arbitrary continuous function. The velocity potential $\varphi$ is thus

$$
\varphi=x A(s)+y B(s)-C(s) .
$$

The functions $A(s)$ and $B(s)$ must be so related that the continuity equation is satisfied. The continuity equation for axially symmetric flow is

$$
\frac{\partial}{\partial x}(\rho u)+\frac{\partial}{\partial y}(\rho v)+\frac{\rho v}{y}=0 .
$$

${ }^{1}$ W. F. Durand, Aerodynamic theory, vol. 3. J. Springer, Berlin, 1935, p. 243.

${ }^{2} \mathrm{H}$. Bateman, Irrotational motion of a compressible inıiscid fluid, Proc. Nat. Acad. Sci., 16, 816 (1930). 
Thus

$$
\left(F A^{\prime}+A F^{\prime}\right) \frac{\partial s}{\partial x}+\left(F B^{\prime}+B F^{\prime}\right) \frac{\partial s}{\partial y}+\frac{F B}{y}=0 .
$$

By Eq. (3), this may be written

$$
\frac{F A^{\prime}+A F^{\prime}}{B^{\prime}}+\frac{F B^{\prime}+B F^{\prime}}{A^{\prime}}=-\frac{F B}{B^{\prime} y \partial s / \partial x}=-\frac{F B}{A^{\prime} y \partial s / \partial y}
$$

unless $B^{\prime}(s) \partial s / \partial x=A^{\prime}(s) \partial s / \partial y=0$. This special case will be discussed later. Except in this special case, Eq. (8) shows that a solution of the type indicated by Eq. (1) is possible only if

$$
y \frac{\partial s}{\partial x}=g(s), \quad y \frac{\partial s}{\partial y}=h(s),
$$

where $g(s)$ and $h(s)$ are arbitrary but related continuous functions of $s$.

The functions $s(x, y)$ which satisfy Eq. (9) can be determined by inverting variables and considering $x=x(s, y)$. Then Eq. (9) becomes (if $\partial s / \partial x \neq 0$ )

$$
\begin{aligned}
& \frac{\partial x}{\partial s}=\frac{1}{\partial s / \partial x}=\frac{y}{g(s)}=y G(s), \\
& \frac{\partial x}{\partial y}=-\frac{\partial s / \partial y}{\partial s / \partial x}=-\frac{h(s)}{g(s)}=H(s) .
\end{aligned}
$$

These expressions may be simply integrated, and it is seen that

$$
x=y H(s)+c,
$$

where $c$ is a constant and $G(s)=H^{\prime}(s)$. Thus the only function $s(x, y)$ which satisfies Eq. (9) is

$$
s=s\left(\frac{y}{x-c}\right)
$$

The excepted case, $\partial s / \partial x=0$, is given by the limit as $c \rightarrow \infty$. If $c$ is finite it may alws ys be made zero by a proper choice of the origin. There are thus only two solutions of Eq. (9),

$$
\begin{aligned}
& s=s(y), \\
& s=s(y / x),
\end{aligned}
$$

The derivation of Eq. (9) breaks down if both sides of Eq. (3) are identically zero. The simplest case occurs if $A^{\prime}(s)=B^{\prime}(s)=0$. This corresponds to a uniform rectilinear flow which is axially symmetric if $B=0$. This is obviously the simplest lost solution and is contained in both Eq. (13) and (14) as $s=s_{0}$, a constant. If $s=s(x)$, both sides of Eq. (3) are identically zero if $B^{\prime}(s)=0$. The continuity equation can then be satisfied only if $B=0$; so this, too, corresponds to a uniform flow. The case, $\partial s / \partial x=0$ and $A^{\prime}(s)=0$, is the limiting case of Eq. (13). This case includes, beside the rectilinear flow along the axis, a two dimension line source on the axis of symmetry or a superposition of the two flows. The remaining case, Eq. (14), leads by Eq. (5) to the Taylor-Maccoll ${ }^{3}$

${ }^{3}$ G. I. Taylor and J. W. Maccoll, Air pressure on a cone moving at high speeds, Proc. Roy. Soc. (A) 139, 278 (1933). 
potential equation and corresponds to the supersonic flow past a cone in the range of Mach numbers for which the shock wave at the nose is attached to the cone. The only lost solutions of the axially symmetric potential flow equation are thus seen to be a rectilinear flow along the axis, a.two dimensional line source on the axis, and the Taylor Maccoll flow past a cone. The first two flows may be superimposed, but the result is of little interest. Only the third conical flow is an essentially three dimensional flow pattern.

In conclusion it can be stated that all the lost solutions of axially symmetric irrotational compressible flow are already well known solutions. This result was apparently known to Bateman; however, it does not appear in any of his writings. A discussion of another class of three dimensional lost solutions, conical flows, has been given by Busemann. ${ }^{4}$

\section{A GENERAL STABILITY CRITERION FOR LINEAR OSCILLATING SYSTEMS WITH CONSTANT TIME LAG*}

\section{By H. I. ANSOFF AND J. A. KRUMHANSL (Brown University)}

Several formulations ${ }^{1}$ have recently been given for the stability criterion of a lumpedparameter linear oscillating system with constant time lag given by the equation

$$
I y^{\prime \prime}(t)+R y^{\prime}(t)+K y(t)=-S y^{\prime}(t-\tau),
$$

where $I, R, K, S$ and $\tau$ are real positive constants and $S y^{\prime}(t-\tau)$ is the feedback term.

By applying Cauchy's index theorem to the operational form of (1) it is possible to generalize the discussion to feedback proportional to any derivative of the dependent variable taken at time $t-\tau$; this will be called a retarded derivative. It is found that the resulting stability criterion can be written in an easily computed form and that the permissible range in the time lag $\tau$ can be stated explicitly.

Equation (1) is generalized to

$$
I y^{\prime \prime}(t)+R y^{\prime}(t)+K y(t)=-S y^{(n)}(t-\tau),
$$

where $n$ is allowed any integer positive value. Letting $F(p)$ be the Laplace transform of $y(t)$, one obtains ${ }^{2}$

$$
F(p)=\frac{L(p)}{Y(p)+S p^{n} e^{-p \tau}}=\frac{L(p)}{Y(p)} \frac{1}{1+S p^{n} e^{-p \tau} / Y(p)},
$$

where

$$
\begin{gathered}
L(p)=I p y(0)+I y^{\prime}(0)+R y(0) \\
Y(p)=I p^{2}+R p+K
\end{gathered}
$$

${ }^{4}$ A. Busemann, Aerodynamischer Auftrieb bei Überschallgeschwindigkeit, Luftfahrtforschung, 12, 210 (1935).

${ }^{*}$ Received Feb. 27, 1948

${ }^{1}$ See references [1] and [2]. In the following, numbers in square brackets refer to the Bibliography at the end of this paper.

${ }^{2}$ The Laplace transform is defined here as in [3]. For other examples of this procedure see reference [4]. 